\title{
THz Double-Grating Gate Transistor Detectors in High Magnetic Fields
}

\author{
D. But, N. Dyakonova, D. Coquillat, F. Teppe, W. Knap \\ Laboratoire Charles Coulomb UMR5221, CNRS and Université Montpellier 2, 34095 Montpellier, France \\ T. Watanabe, Y. Tanimoto, S. Boubanga Tombet and T. Otsuji \\ Research Institute of Electrical Communication, Tohoku University, 2-1-1 Katahira, Aoba-Ku \\ Sendai 980-8577, Japan

\begin{abstract}
Double-grating-gate field-effect transistors have a great potential as terahertz detectors. This is because the double grating gate serves not only for carrier density tuning but also as an efficient $\mathrm{THz}$ radiation coupler. In this paper, we present characterization of these transistors using high magnetic fields. Low and high magnetic field data are used to determine the electron mobility and electron concentration, respectively, in different parts of the transistor channel.
\end{abstract}

PACS: 07.57.Kp, 85.30.Tv, 73.43.Qt

\section{Introduction}

Detection of terahertz $(\mathrm{THz})$ radiation by an asymmetric unit cell double-grating-gate field-effect transistor (A-DGG-FET) at room temperature was theoretically studied in papers [1, 2]. In Refs. [3, 4] there was demonstrated high photovoltaic responsivity exceeding $2.2 \mathrm{kV} / \mathrm{W}$ at $1 \mathrm{THz}$ with a low noise equivalent power of $15 \mathrm{pW} / \mathrm{Hz}^{1 / 2}$ at room temperature. Typical structure of the A-DGG-FET with an asymmetric unit cell is shown in Fig. 1. Two grating gates with different widths were formed with the $\mathrm{Ti} / \mathrm{Au} / \mathrm{Ti}$ gate-to-channel separation $d=65 \mathrm{~nm}$. The theoretically assumed values of carrier density $N^{(0)}$ and scattering time $s$ were: $N^{(0)}=2.5 \times 10^{12} \mathrm{~cm}^{-2}$ and $s=2.9 \times 10^{-13} \mathrm{~s}$ (which corresponds to a room temperature electron mobility $12,000 \mathrm{~cm}^{2} /(\mathrm{V} \mathrm{s})$ in an InAlAs/InGaAs/InP heterostructure) $[1,4]$.

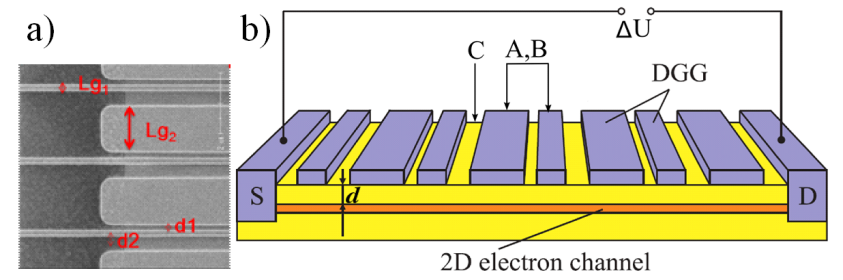

Fig. 1. The photo (a) and schematic (b) view of the double-grating-gate FET structure with an asymmetric unit cell. External $\mathrm{THz}$ wave is incident normally from the top. The parameters of the asymmetric structure are $\mathrm{Lg}_{1 \min }=400 \mathrm{~nm}, \mathrm{Lg}_{1 \max }=705 \mathrm{~nm}, \mathrm{Lg}_{2}=$ $1600 \mathrm{~nm}, d 1=400 \mathrm{~nm}, d 2=800 \mathrm{~nm}[4]$.

\section{Experiments}

The photovoltaic detection signal was investigated as a function of the gate voltage and magnetic field using an InGaP/InGaAs/GaAs A-DGG-FET structure. The incident radiation of $0.3 \mathrm{THz}$ was provided by a Gunn diode which was modulated by a chopper at frequency $576 \mathrm{~Hz}$.

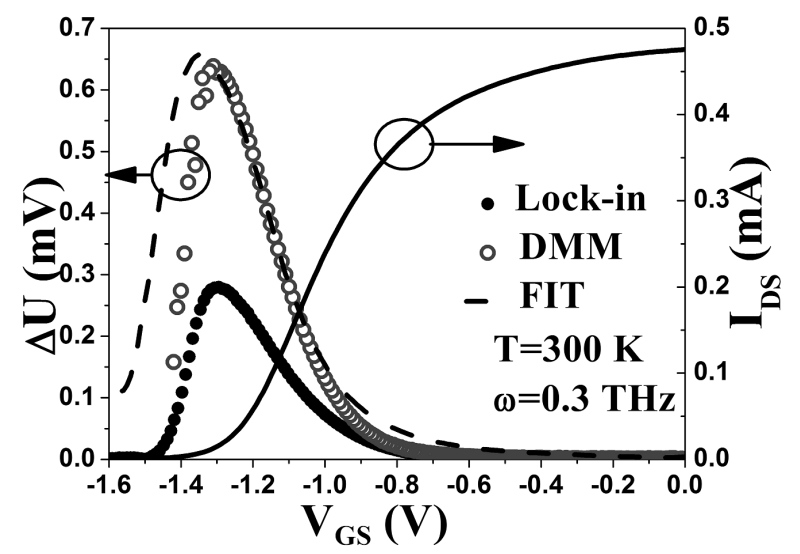

Fig. 2. Characteristics of the detector as a function of gate bias $V_{\mathrm{gs}} \mathrm{DC}$ transfer characteristics $I_{\mathrm{DS}}\left(V_{\mathrm{GS}}\right)$ (right $y$-axis, black line) and measured (points) and the calculated (Eq. (1)), dashed-lines) photovoltages $\Delta U\left(V_{\mathrm{GS}}\right)$ at $0.3 \mathrm{THz}$ (left $y$-axis).

The photovoltaic detection signals were registered by different methods (see Fig. 2) showing importance of loading effects [5]. The parasitic read-out circuit capacitances were determined by independent measurement 
with an RLC bridge and found to be $\approx 150 \mathrm{pF}$ for lock-in amplifier technique. To measure the amplitude of the signal, the Keithley source-meter was used as a current source with a parallel voltmeter. This method can virtually eliminate the internal parasitic resistance of the read-out circuit. These results correspond to the overdamped plasma detection case (broadband detection) described in Ref. [6]. Dashed line in Fig. 2 fits well the experimental results using Eq. (1).

Theory in Ref. [5] corresponds to the broadband detection signal $\Delta U$ with the channel conductivity $\sigma\left(V_{\mathrm{GS}}\right)$. The resulting simple formula can be written as follows:

$$
\Delta U=\frac{U_{\mathrm{a}}^{2}}{4}\left[\frac{1}{\sigma} \frac{\mathrm{d} \sigma}{\mathrm{d} V_{\mathrm{GS}}}\right]_{V_{\mathrm{GS}}=V_{\mathrm{GS}_{0}}},
$$

where $U_{\mathrm{a}}$ is the amplitude of the AC gate-to-source voltage induced by the incident $\mathrm{THz}$ radiation (it reflects the antenna coupling efficiency and the power of the $\mathrm{THz}$ source), $V_{\mathrm{GS}}$ is averaged by time and the channel length voltage swing $V_{\mathrm{GS}}=V_{\mathrm{g}}-V_{\mathrm{th}}\left(V_{\mathrm{th}}\right.$ is the threshold voltage).

\section{Results}

The wavelength of $0.3 \mathrm{THz}$ (integral power of source is $3 \mathrm{~mW}$ ) radiation corresponds to $1 \mathrm{~mm}$, which is larger than the transistor's active area $\left(20 \times 20 \mu \mathrm{m}^{2}\right)$. The incident radiation is coupled to the channel via the contact pads playing the role of antennas (see Fig. 3a). These contact pads are symmetric with respect to the transistor channel and comparable with the incident wavelength ( $L=2.5 \mathrm{~mm}$ ). The broadband rectification signal is obtained with the combined actions of the carrier velocity and density nonlinearities in the channel, and of the source-to-drain asymmetry. Therefore, without source-to-drain asymmetry, the rectification signal is linked to the position of the $\mathrm{THz}$ spot on the contact pads. If the spot is centered on the middle of the device, then the signals generated at the drain and source sides lead to a compensating dc voltage (rectification at the both side are equal) that decreases the responsivity dramatically. In the opposite case, if the spot is shifted on one side of the structure, the rectified signal is maximal. Figure $3 \mathrm{~b}$ corresponds to the image of the $\mathrm{THz}$ spot, showing the influence of the antenna on the photoresponse. One can clearly see two black and white regions on the raster scan image, corresponding to the sign of the measured photoresponse as a function of the position of the spot onto the structure. Figure $3 \mathrm{c}$ shows the amplitude and the sign of the photoresponse signal as a function of the spot position onto the structure. On the left side the signal is negative, in the middle of the device the signal turns to zero and changes its sign to become positive on the right side of the device.

Further experimental studies of photoresponse and magnetoresistance were carried out at liquid helium temperatures and magnetic field up to $15 \mathrm{~T}$. The measured $\mathrm{THz}$ photoresponse revealed a pronounced oscillating behavior as a function of inverse magnetic field (see Fig. 4).



Fig. 3. (a) The A-DGG-FET detector with contact pads. (b) The influence of symmetric metallic pads (antenna) on the photoresponse; (c) the amplitude and the sign of the photoresponse signal as a function of the spot position onto the structure.

This is a consequence of relatively high carrier mobility in device channel which was around $6,000 \mathrm{~cm}^{2} /(\mathrm{Vs})$ at room temperature. The experimental value of mobility is lower than the theoretical value due to the effect of transistors channel geometry.

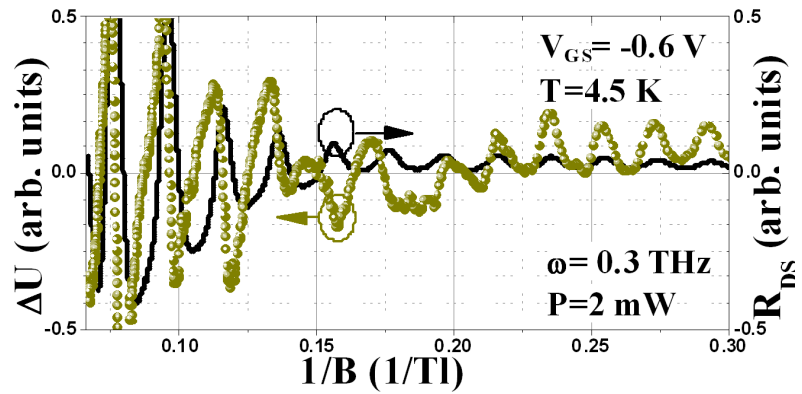

Fig. 4. Comparison of the magnetoresistance and $0.3 \mathrm{THz}$ photoresponse oscillations as a function of the magnetic field for gate voltage $-0.6 \mathrm{~V}$.

The electron concentration in the gated part of transistor channel was determined from high $B$ part of photoresponse by the Fourier transform of Shubnikov-de Haas oscillations plotted as a function of $B^{-1}[3]$. Power spectra of the Fourier transform corresponding to signal $\Delta U$ and channel resistance $R_{\mathrm{DS}}$ of Fig. 4 are presented in Fig. 5a. One can observe two main maxima in the 


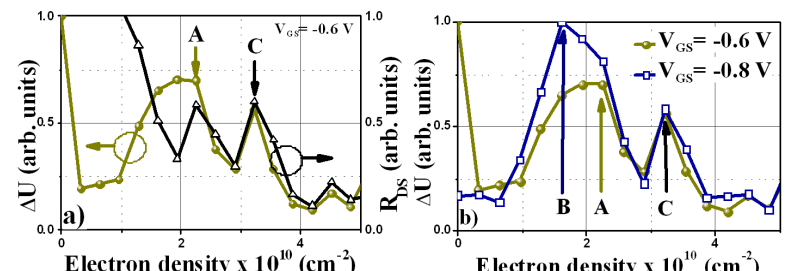

Fig. 5. Power spectra of Fourier transform: (a) from photoresponse (circle) and magnetoresistance (triangle) at $V_{\mathrm{GS}}=-0.6 \mathrm{~V}$; (b) the concentration swing at different gate voltage.

power spectra that are marked as $A$ and $C$. We tentatively attribute these maxima to two regions of the channel. The ungated part and the gated part correspond to maxima $C$ (carrier density $3.22 \times 10^{10} \mathrm{~cm}^{-2}$ ) and $A$ $\left(2.25 \times 10^{10} \mathrm{~cm}^{-2}\right)$, respectively, as shown in Fig. 1 . The gated part maxima in the power spectra changed position when the gate voltage changes, the concentration in the ungated part remained unchanged (the shift from point $A$ to $B$ ), as shown in Fig. 5b. These data are in rough agreement with parameters assumed in the first theoretical estimates. They will allow for future more precise calculations of the THz photoresponse of A-DGG-FET structures.

\section{Conclusion}

It was shown that FETs with a A-DGG-FET structure can operate at room temperature as sensitive broadband sub- $\mathrm{THz}$ detectors. When the incident radiation frequency is sufficiently low (wavelength larger than device active area), it was also shown that the incident $\mathrm{THz}$ radiation is coupled to the device through its contact pads.
Without asymmetry of the contact pads/antennas, the rectification signal was shown to be related to the position of the spot onto the contact pads. Finally, it was shown under magnetic field, in the conditions when both gates are equally biased, that the double grating gates are acting as a unique and large gate on the channel.

\section{Acknowledgments}

This work was performed in the frame of French Japanese JST-ANR project WITH.

\section{References}

[1] V.V. Popov, D.V. Fateev, T. Otsuji, Y.M. Meziani, D. Coquillat, W. Knap, Appl. Phys. Lett. 99, 243504 (2011).

[2] V.V. Popov, D.V. Fateev, T. Otsuji, Y.M. Meziani, D. Coquillat, W. Knap, in: Int. Poster, Intern. TeraNanoEGGDRI Workshop Extended Abstract, Osaka, 2011.

[3] T. Watanabe, S. Boubanga Tombet, Y. Tanimoto, Y. Wang, H. Minamide, H. Ito, D. Fateev, V. Popov, D. Coquillat, W. Knap, T. Otsuji, in: ISDRS 2011: Intern. Semiconductor Device Research Symp. Digest, FP3-06, Univ. Maryland, MD, USA, 2011.

[4] T. Watanabe, S.B. Tombet, Y. Tanimoto, Y. Wang, H. Minamide, H. Ito, D. Fateev, V. Popov, D. Coquillat, W. Knap, Y. Meziani, T. Otsuji, Solid-State Electron. 78, 109 (2012).

[5] M. Sakowicz, M.B. Lifshits, O.A. Klimenko, F. Schuster, D. Coquillat, F. Teppe, W. Knap, J. Appl. Phys. 110, 054512 (2011).

[6] W. Knap, V. Kachorovskii, Y. Deng, S. Rumyantsev, J.Q. Lu, R. Gaska, M.S. Shur, G. Simin, X. Hu, M.A. Khan, C.A. Saylor, L.C. Brunel, J. Appl. Phys. 91, 9346 (2002). 